\title{
The behaviour of heavy particles in the expanding plasma jet in argon
}

\author{
M C M van de Sanden, J M de Regt and D C Schram \\ Department of Physics, University of Technology Eindhoven, PO Box 513, \\ 5600 MB Eindhoven, The Netherlands
}

Received 11 February 1993, in final form 24 November 1993

\begin{abstract}
This paper concerns the behaviour of heavy particles in an argon plasma expanding from a cascaded arc. The plasma is characterized using high-accuracy spatially resolved Thomson-Rayleigh measurements. It is shown that the expansion of the heavy particles, neutral particles and ions, is close to adiabatic and that three-particle recombination has a small effect on the ion density. The measurements are compared with an adiabatic model and are found to be in good agreement. The behavio dr of the neutral particles in the shock front is independent from that of the ions. The neutral particle shock front is identical to the shock front found in neutral gases and is accordingly characterized by the ne $t$ tral particle Mach number. It is experimentally confirmed that the shape of the shock front is of the Mot-Sm th: type. The motion of the ions in the shock front is influenced by the presence of the electrons in such a way that the electrons are compressed by the ions. The shape of the ion shock front is of the Mott-Smith type, however, with a different Mach number which includes the electron temperature.
\end{abstract}

\section{Introduction}

Expanding plasmas have become more and more interesting from both a technological point of view as well as from a more fundamental point of view. These types of plasma are used in the recently developed fast deposition method (up to a factor of hundred higher rates than with the conventional deposition methods) for several types of carbon coatings (ranging from crystalline graphite and diamond to amorphous hydrogenated carbon the so called 'diamond like coatings') and amorphous hydrogenated silicon layers $[1-3]$. Also the use of these type of plasmas for bright atomic and ionic hydrogen sources is presently under investigation [4]. Furthermore, these type of plasmas are used in thrusters for space applications [5].

For the applications mentioned a detailed fundamental insight into the expanding plasma is necessary. For example, the recently reported drastic decrease of the electron density in argon/hydrogen plasmas [4], for even small amounts of hydrogen admixtures, makes a detailed study of the dynamics of these types of plasmas necessary. Moreover, since in most applications for plasma deposition argon is the carrier gas, a detailed study of a pure expanding argon plasma is essential. Phenomena observed in argon/hydrogen plasmas or with additional admixtures of methane $\left(\mathrm{CH}_{4}\right)$ or silane $\left(\mathrm{SiH}_{4}\right)$ can then be related to the pure argon case.

In this paper the fundamental aspects of a plasma expanding from a cascaded arc are discussed using accurate spatially resolved Thomson--Rayleigh scatter- ing measurements. In the next section the experimental set-up and the Thomson-Rayleigh scattering diagnostics are shortly discussed. The succeeding sections concern with the discussion of the performed measurements and the comparison with different models reported in the literature. Finally conclusions will be given.

\section{Experiment}

The measurements discussed in this paper are performed on a plasma which expands freely from a sub atmospheric thermal plasma in argon (a cascaded arc, typical values for temperature $T \approx 1 \mathrm{eV}$, electron density $n_{\mathrm{e}} \approx$ $10^{22} \mathrm{~m}^{-3}$ and neutral particle density $n_{0} \approx 10^{23} \mathrm{~m}^{-3}$, see figure 1$)$ into a low background pressure $(p \approx 10$ $100 \mathrm{~Pa}$ ) [6-9]. The plasma has been described in detail elsewhere [7]. The settings discussed in this paper are listed in table 1. The electron density and temperature and the neutral particle density in the expansion part are measured locally by means of Thomson and Rayleigh scattering. The main components of the scattering diagnostic are: a frequency doubled Nd: YAG laser, a polychromator based on a holographic concave grating and a gateable light amplifier in combination with a linear photodiode array. Much attention is given to the suppression of stray light which is essential if one wants to measure Rayleigh and Thomson scattering simultaneously (equivalent stray light level $0.4 \mathrm{~Pa}$ argon at $300 \mathrm{~K}$ ). One of the main advantages of ThomsonRayleigh scattering is that the spatially resolved values 


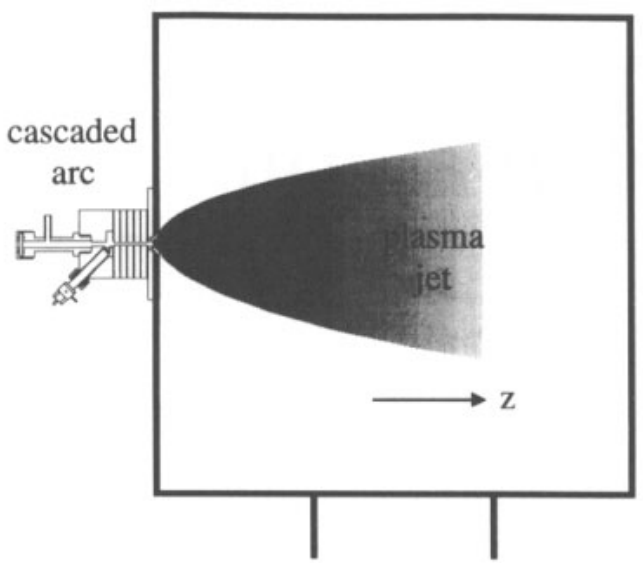

to pumps

Figure 1. The expanding cascaded arc. Schematically, to the left a cascaded arc is attached to a vacuum chamber, the plasma jet expands into a low background pressure. The exit of the cascaded arc corresponds to the $z=0$ position.

of $n_{\mathrm{e}}, T_{\mathrm{e}}$ and $n_{0}$ are measured, i.e. no Abel inversion has to be performed. For more details concerning the Thomson-Rayleigh scattering diagnostic, the reader is referred to the original publication [7].

\section{Results and discussion}

\subsection{Thomson-Rayleigh scattering results}

In figures 2 and 3 the measured $n_{\mathrm{e}}, n_{0}$ and $T_{\mathrm{e}}$, on the axis of the plasma jet, as a function of the axial position $z$, for the five settings as given in table 1 , are shown. Setting 1 of table 1 is also shown in figure 3 for comparison. The position of $z=0 \mathrm{~mm}$ corresponds to the exit of the cascaded arc. Note the accuracies in $T_{e}$ and $n_{0}$ for the lowest background pressure. The accuracy of the neutral particle density for values below $7 \times 10^{19} \mathrm{~m}^{-3}$ is about $100 \%$, i.e. these values are at the detection limit. The accuracy in $T_{\mathrm{e}}$ just behind the density shock front is for $p=13.3 \mathrm{~Pa}$ worse than for $p=40$ and $133 \mathrm{~Pa}$ (see figure 2 ). The reproducibility of the measurements was checked by performing more measurements for a certain position during the day. All the measurements showed that the reproducibility was within the estimated error. The accuracy in $T_{e}$ is related to the electron density: the accuracy is better for larger electron densities. For $n_{\mathrm{e}} \approx 3 \times 10^{18} \mathrm{~m}^{-3}$ the error in $T_{\mathrm{e}}$ is approximately 10 to $20 \%[7,8]$.

Table 1. The different settings of the cascaded arc set-up. For explanation see text.

\begin{tabular}{llll}
\hline Settings & larc $_{\text {a }}$ & Ar flow $\left(\operatorname{scc~s}^{-1}\right)$ & $p(\mathrm{~Pa})$ \\
\hline 1 & 45 & 58 & 40 \\
2 & 45 & 58 & 13.3 \\
3 & 45 & 58 & 133 \\
4 & 45 & 75 & 40 \\
5 & 60 & 58 & 40 \\
\hline
\end{tabular}

(a)

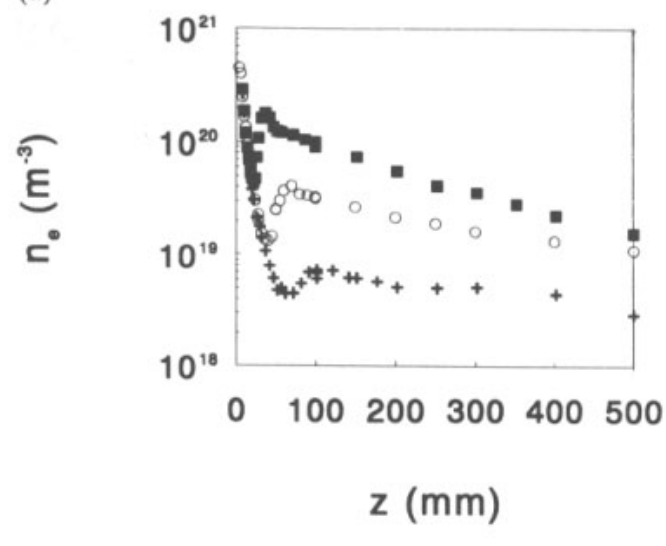

(b)

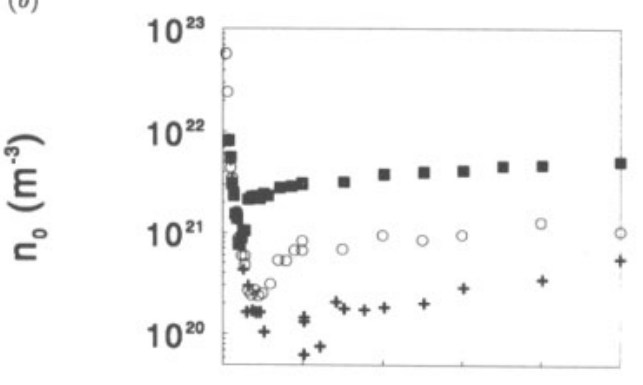

0100200300400500

$\mathbf{z}(\mathrm{mm})$

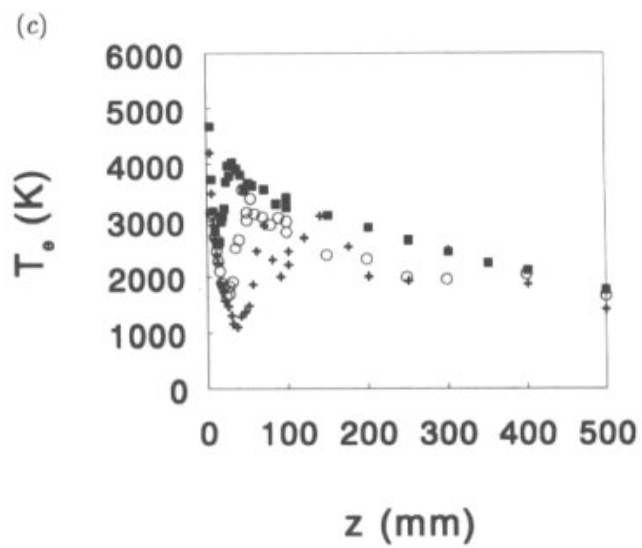

Figure 2. $n_{\mathrm{\theta}}(a), n_{0}(b)$ and $T_{\mathrm{e}}(c)$ on the axis as a function of the axial position for different background pressures. $l_{\text {arc }}=45 \mathrm{~A}$; Ar flow $=58 \mathrm{scc} \mathrm{s}^{-1}$; $+13.3 \mathrm{~Pa}$; O, 40 Pa; घ, $133 \mathrm{~Pa}$.

The division of the plasma into the supersonic expansion, the stationary shock front and the subsonic relaxation region (the region behind the shock front) is clearly seen in figures $2(a)$ and $(b)$ and $3(a)$ and $(b)$. As known from measurements and calculations in the cascaded arc [9], for equivalent settings as given in table 1 , 
(a)

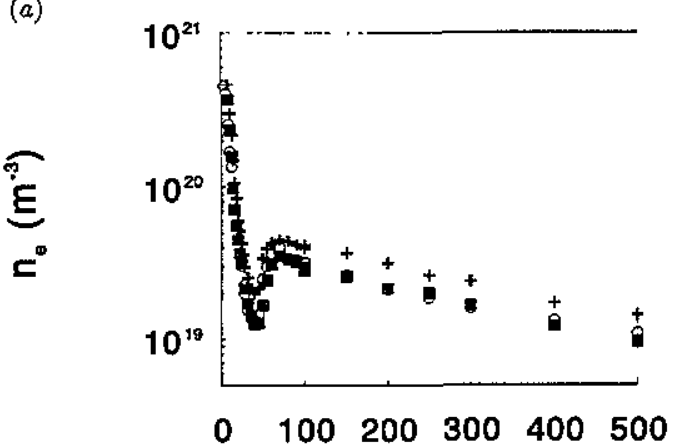

$z(\mathrm{~mm})$

(b)

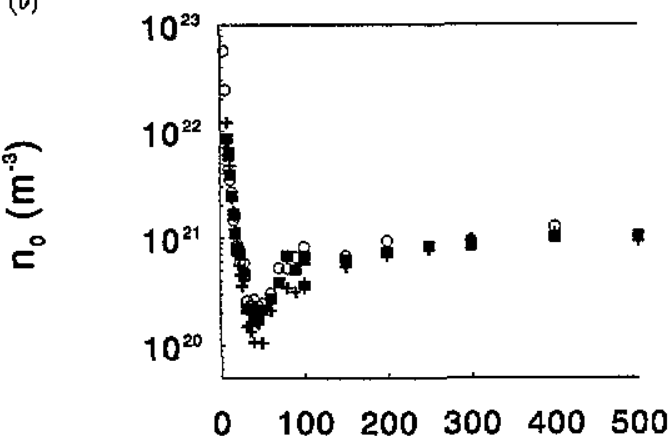

$z(\mathrm{~mm})$

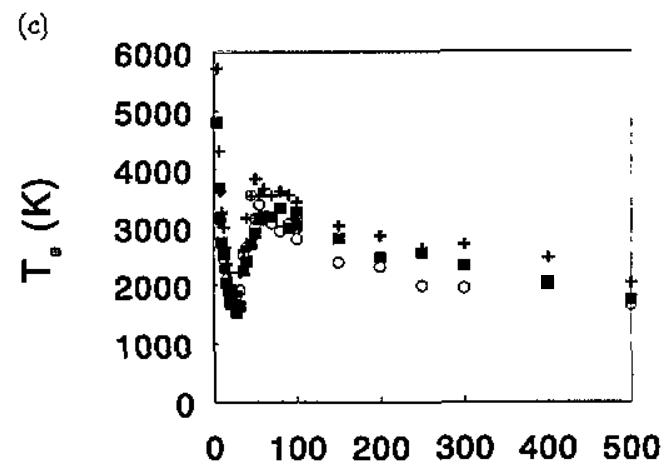

$z(\mathrm{~mm})$

Figure 3. $n_{\mathrm{e}}(a), n_{0}(b)$ and $T_{\mathrm{e}}(c)$ on the axis as a function of the axial position for different settings of the cascaded arc for a vessel pressure $p=40 \mathrm{~Pa} ; O, I_{\text {arc }}=45 \mathrm{~A}$ and $\mathrm{Ar}$ flow $=58$ $\operatorname{scc~s}^{-1} ;+, l_{\text {arc }}=60 \mathrm{~A}$ and Ar flow $=58 \mathrm{scc} \mathrm{s}^{-1} ; \mathbf{\square}, l_{\text {arc }}=$ $45 \mathrm{~A}$ and $\mathrm{Ar}$ flow $=75 \mathrm{scc} \mathrm{s} \mathrm{s}^{-1}$.

the values of the electron and neutral particle densities at the exit of the cascaded arc are approximately equal to $n_{\mathrm{e}} \approx 10^{22} \mathrm{~m}^{-3}$ and $n_{0} \approx 10^{23} \mathrm{~m}^{-3}$. This means that in the first part of the expansion the densities decrease by three orders of magnitude. Because the electron density is equal to the ion density, the measurement of the electron density gives information on the dynamic behaviour of the ions as well. This is related to the fact that it is easier to estimate the divergence of the ion flux. Therefore, in the treatment of the mass balance for the electron or ion density, it is more instructive to discuss the properties in terms of the ion dynamics. The decrease of the electron temperature in the expansion region is smaller than is expected on the basis of an adiabatic expansion. In that case the temperature would decrease as $n_{\mathrm{e}}^{2 / 3}$, which means for the situation of the setting 1 of table 1 (see figure 2) that the electron temperature would decrease by two orders of magnitude. The reason for this diabatic behaviour in the expansion of the electron gas will be discussed in the next section. As mentioned a shock occurs both in the ion and in the neutral particle density. The difference in shock front thickness for the neutral particles and ions is related to the mean free paths of the ions and neutral particles ahead of the shock. As can be seen from figures 2 and 3 , the jump in electron temperature occurs in front of the jump in densities. The structure and thickness of the shock front will be treated in more detail later on. A separate paper is devoted to the heating mechanism of the electron gas in the stationary shock front [10]. After the shock front, the electron density decreases slowly. The neutral particle density increases towards the background value.

It is clear from figures $2(a)$ and $(b)$ that the background pressure has no effect on the first part of the expansion. However, the start of the shock fronts, as measured from the exit of the cascaded arc, advances if the background pressure decreases. As in Young's [11] work, it is observed that the position of the shock front is found at larger distances from the cascaded arc exit, if the background pressure is lowered.

By changing the flow $Q$ and the arc current $I_{\text {arc }}$ (see table 1), the values of the velocity, the temperature, and in particular the densities for the different species, are different at the exit of the cascaded arc. As can be seen from figure $3(a) n_{e}$ is larger if $I_{\text {arc }}$ is changed from $45 \mathrm{~A}$ to $60 \mathrm{~A}$. For a higher flow in the cascaded arc, the results for the electron density are similar to setting 1 of table 1. The neutral particle density is lower for a higher current in the cascaded arc, leading to a higher ionization degree. This is probably related to the fact that for a higher current the neutral particle density profile in the cascaded arc is different from setting 1 of table 1 , which affects the neutral particle density profile in the expansion. For the higher flow in the cascaded arc, the neutral particle density in the expansion is approximately equal to setting 1 of table 1 . The differences in the electron temperature are small for the different settings in the cascaded arc. For the situation with $I_{\text {arc }}=60 \mathrm{~A}$ the electron temperature is, however, systematically larger than for the other four settings. The difference in electron temperature is especially clear behind the electron temperature jump and in the subsonic relaxation region.

In figures $4(a)-(c)$ the electron and neutral particle 

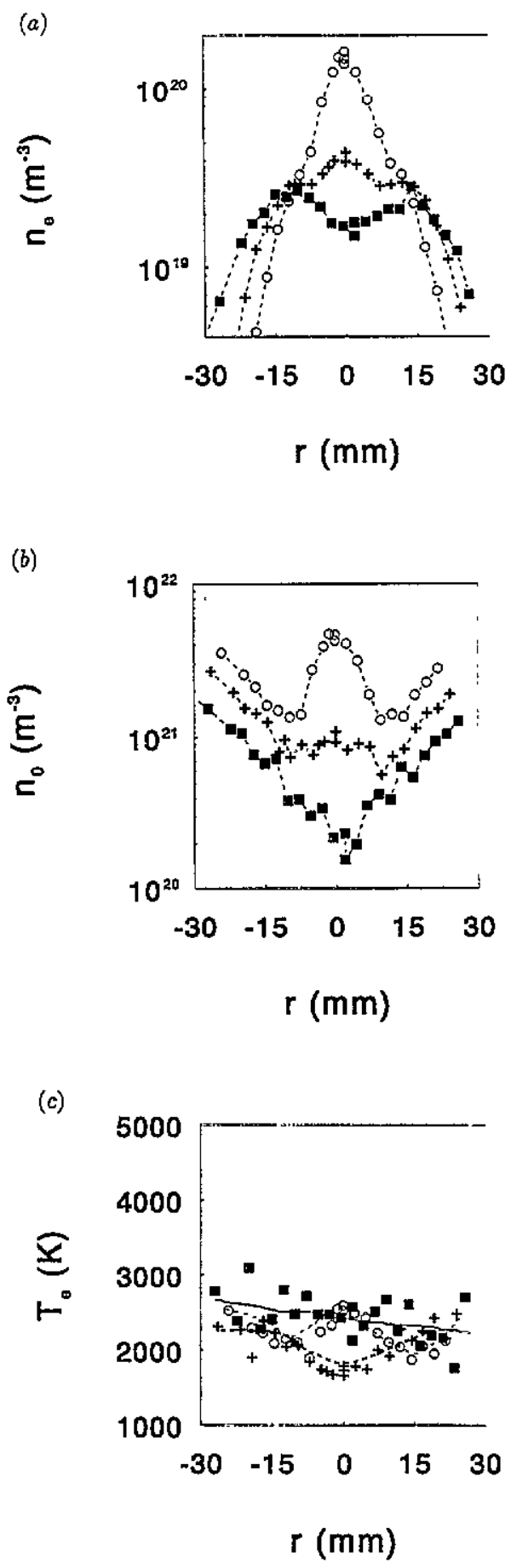

Figure 4. $n_{e}(a), n_{0}(b)$ and $T_{e}(c)$ as a function of the radial position for three axial positions: $O, z=10 \mathrm{~mm} ;+, z=$ $20 \mathrm{~mm} ; \mathbf{u}, z=40 \mathrm{~mm}$.

density and the electron temperature as a function of the radial coordinate $r$ are shown for $z=10 \mathrm{~mm}, z=20 \mathrm{~mm}$ and $z=40 \mathrm{~mm}$ for setting 1 of table 1 . As is seen from figure 4 , in the expansion, a structure corresponding to the so called barrel shock is present [12], resulting in two dips in the neutral particle density. The electron density has a dip just ahead of the shock front. Close to the exit of the cascaded arc, the radial profile of the electron density has a small peak on top of a broader profile. In figure 5 the Thomson-Rayleigh scattering measurements are shown in a smoothed three-dimensional representation which shows that, after the shock
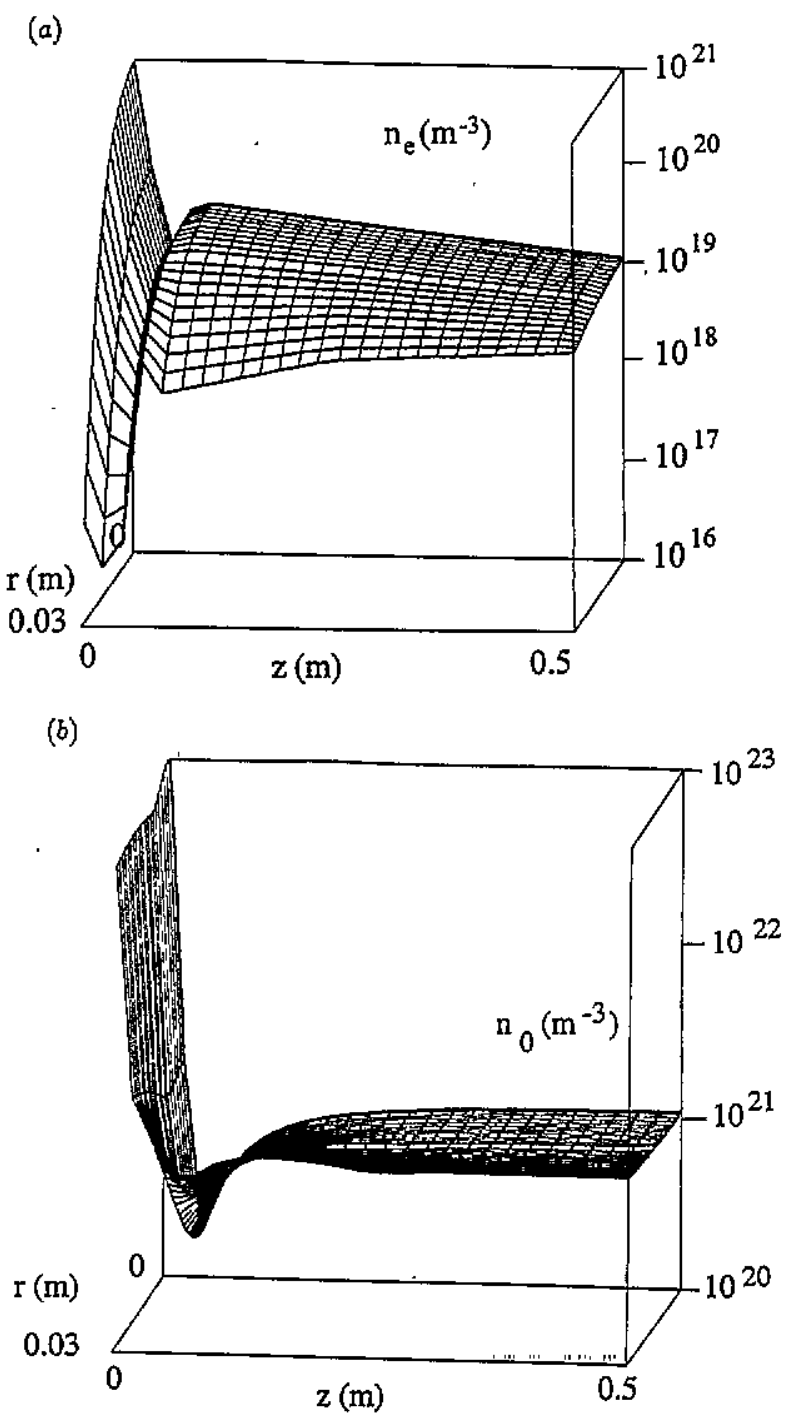

(c)

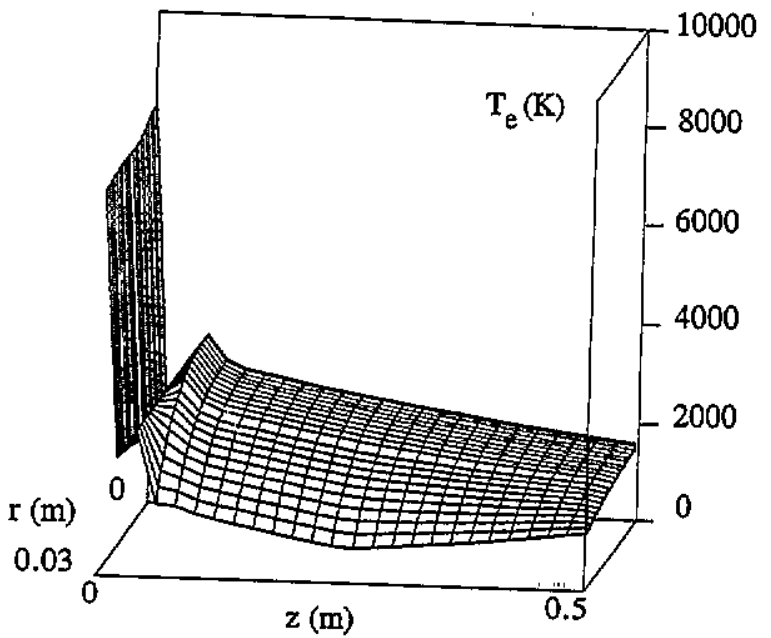

Figure 5. A smoothed three-dimensional representation of the electron density $n_{\mathrm{e}}(a), n_{0}(b)$, and $T_{\mathrm{e}}(c)$. 
front, the structure in the radial profiles of the electron density and temperature and the neutral particle density disappears. Furthermore, the typical structure of the shock front can be seen clearly.

The results presented in figures 2 and 3 are similar to results obtained previously $[13-16]$. The radial profiles given in figure 4 are similar to the results of Kobayashi et al [17], who measured the densities in the barrel shock in a expanding plasma jet using interferometry.

In the next two sections 3.2 and 3.3 the supersonic expansion and the shock front in the densities will be treated in more detail.

\subsection{The supersonic expansion}

Figure 6 shows the measured behaviour of $n_{0}, n_{\mathrm{e}}\left(\approx n_{\mathrm{i}}\right)$ as a function of $z$ in the expansion together with the relation derived by Ashkenas and Sherman [12]. This model was originally developed for the adiabatic super-
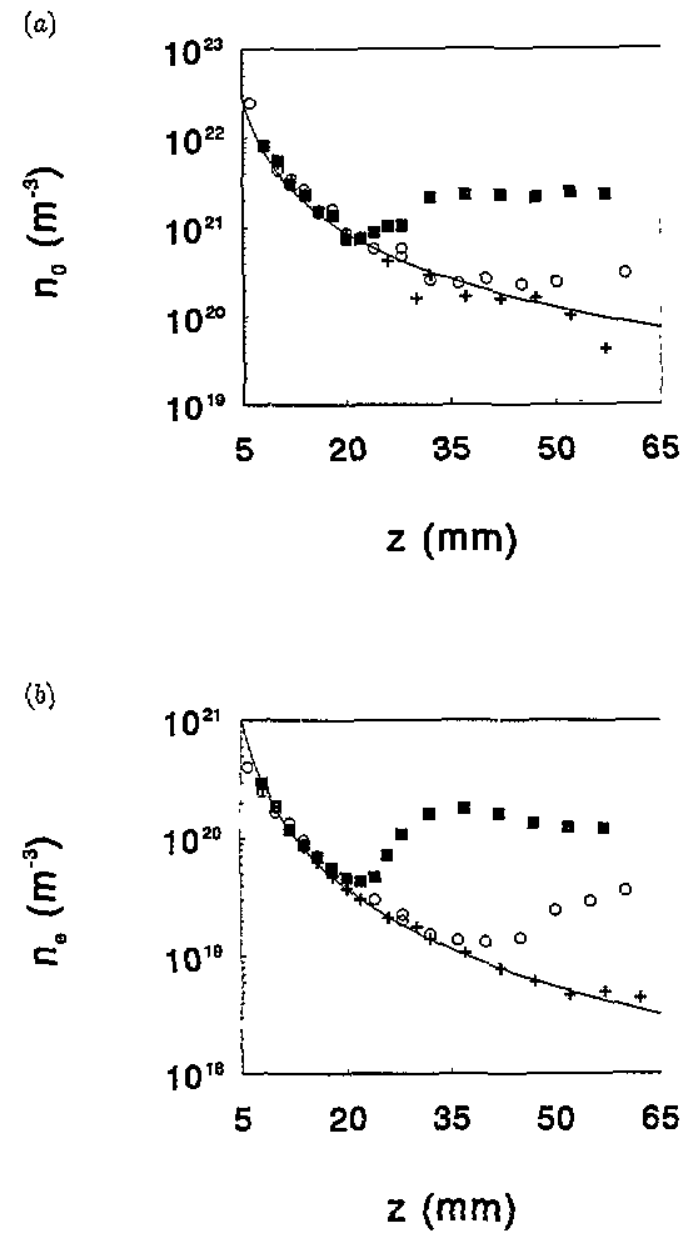

Figure 6. $n_{0}(a)$ and $n_{\mathrm{e}}(b)$ on the axis as a function of the axial position for different background pressures compared with the model of Ashkenas and Sherman (curves) [12]. $l_{\text {arc }}=45 \mathrm{~A}$; Ar flow $=58 \mathrm{scc} \mathrm{s}^{-1} ;+13.3 \mathrm{~Pa} ; 0,40 \mathrm{~Pa}$; I, $133 \mathrm{~Pa}$. sonic expansion of ideal gases. Following this model the densities scale as

$$
n=\frac{n_{\text {res }} z_{\text {ref }}^{2}}{\left(z-z_{0}\right)^{2}}
$$

for $z \gg z_{0}$. Equation (1) is the well known source expansion with $n_{\text {res }}$ the reservoir particle density, $z_{0}$ the origin from which the particle trajectories seem to originate and $z_{\text {ref }}$ a reference length. As can be seen from figure 6 the agreement of equation (1) with the measurements of $n_{0}$ and $n_{\mathrm{e}}$ is excellent. The interpretation of equation (1) is as follows. During the expansion, thermal energy is converted adiabatically into directed kinetic energy. Since the thermal energy is limited by the total enthalpy in the reservoir (in the present case the conditions at the end of the cascaded arc), the velocity $w_{\text {axis }}$ along the axis saturates and becomes finally constant. From flux conservation this means that for a source expansion the product $n w_{\mathrm{axis}} A=$ constant. Since the area $A$ of the plasma jet scales as $z^{2}$, the densities scale as $z^{-2}$ for large $z$ (see equation (1)). For a plasma, however, equation (1) is only valid if the recombination or ionization can be neglected compared with the change of density due to the expansion. In table 2 the parameters $z_{0}$ and $n_{\text {res }}$, corresponding to the different settings of table 1 , are given. As can be seen from table 2, for the same settings of the plasma, the parameters $z_{0}$ and $n_{\text {res }}$ for the electron and neutral particle density are the same within the estimated error. This means that the change of the ionization degree during expansion of the plasma is minimal. Another conclusion is that for the same arc settings but different vessel pressure the fitting parameters are equal, indicating the supersonic nature of the expansion. Hence, from table 2 it can be concluded that the recombination of argon during the expansion is much smaller than the decrease of the densities caused by the expansion of the plasma.

An alternative approach to determine the recombination of argon in an expanding plasma jet has been used, i.e. by means of the electron energy balance [18]. For $n_{\mathrm{e}}$ as a function of $T_{\mathrm{e}}$ for the plasma settings as given in table 1 , a simple scaling law is found which reads

$$
T_{\mathrm{e}}^{x}=C_{\mathrm{o}} n_{\mathrm{e}}
$$

The parameters $\chi$ and $C_{0}$ are determined from a weighted least-mean square analysis and are equal to $\chi=3.47 \pm 0.26$ and $C_{0}=4.7 \times 10^{-9} \mathrm{~m}^{3} \mathrm{~K}^{3.47}$. The

Table 2. The fitting parameters $z_{0}$ and $n_{\text {res }}$ for the electron and the neutral particle density in the expansion.

\begin{tabular}{lllll}
\hline Settings & $z_{0, \mathrm{e}}(\mathrm{mm})$ & $\begin{array}{l}n_{\text {res, }} \\
\left(10^{22} \mathrm{~m}^{-3}\right)\end{array}$ & $z_{0,0}(\mathrm{~mm})$ & $\begin{array}{l}n_{\text {res }, 0} \\
\left(10^{23} \mathrm{~m}^{-3}\right)\end{array}$ \\
\hline 1 & $1.5 \pm 0.5$ & $1.3 \pm 0.3$ & $1.5 \pm 0.5$ & $3.0 \pm 0.3$ \\
2 & $1.5 \pm 0.5$ & $1.3 \pm 0.3$ & $1.5 \pm 0.5$ & $3.0 \pm 0.3$ \\
3 & $1.5 \pm 0.5$ & $1.3 \pm 0.3$ & $1.5 \pm 0.5$ & $3.0 \pm 0.3$ \\
4 & $2.5 \pm 0.5$ & $1.5 \pm 0.3$ & $2.5 \pm 0.5$ & $3.0 \pm 0.3$ \\
5 & $3.5 \pm 0.5$ & $1.7 \pm 0.3$ & $3.5 \pm 0.5$ & $2.0 \pm 0.3$ \\
\hline
\end{tabular}


accuracy in $C_{0}$ is approximately a factor three. Since $\chi>\frac{3}{2}$ the electron gas is heated in the expansion. Using a quasi one-dimensional model and equation (2), it was shown analytically that this heating is caused by threeparticle recombination [18]. The absolute value of the recombination coefficient determined, with the familiar $T_{e}^{-9 / 2}$ dependence [19], was within a factor of three equal to the values found in the literature $[20,21]$.

\subsection{The shock front}

Before we discuss the features of the measured shock front in the neutral particle and ion density, we will first shortly review some of the shock theory available in the literature.

The shock front is an example of a surface of discontinuity in gas dynamics [22]. In the situation of a discontinuity, the flow parameters such as the temperature, density, pressure and velocity, on each side of the discontinuity surface are given by relations which describe the conservation of mass, momentum and energy. For a perfect gas with $\gamma=\frac{5}{3}\left(\gamma=c_{p} / c_{v}\right.$ with $c_{p}$ and $c_{v}$ the specific heat at constant pressure and volume), these jump relations read $[22,23]$

$$
\begin{gathered}
\frac{\rho_{0,2}}{\rho_{0,1}}=\frac{w_{0,1}}{w_{0,2}}=\frac{4 M_{0,1}^{2}}{M_{0,1}^{2}+3} \\
\frac{T_{0,2}}{T_{0,1}}=\frac{\left(5 M_{0,1}^{2}-1\right)\left(M_{0,1}^{2}+3\right)}{16 M_{0,1}^{2}}
\end{gathered}
$$

where $\rho_{0}, w_{0}$, and $T_{0}$ are the mass density, the velocity and the temperature of the neutral gas. The subscripts 1 and 2 refer to the quantities ahead of and behind the shock front respectively. Note that when $M_{0,1} \rightarrow \infty$ the compression ratio (equation (3)) approaches (4). Equations (3) and (4) are equivalent to the RankineHugoniot relations [23]. Here $M_{0}$ is the Mach number defined as

$$
M_{0}=\frac{w_{0}}{\sqrt{\frac{5}{3} \Re T_{0}}}
$$

with $R$ the gas constant for argon given by $k_{\mathrm{b}} / m_{\mathrm{b}}\left(k_{\mathrm{b}}\right.$ is the Boltzmann constant and $m_{h}$ the mass of the argon atom). Through the shock front, the supersonic flow transforms into a subsonic flow. The thickness of a shock front for a perfect gas is of the order of the mean free path for neutral-neutral collisions ahead of the shock front $\lambda_{00,1}[22,23]$. In the kinetic approach by Mott-Smith [24] and later extended by several others $[25,26]$, the solution for the ratio of the densities, can be written as

$$
\rho_{0}(z)=\rho_{0,1}+\frac{3 \rho_{0,1}\left(M_{0,1}^{2}-1\right)}{\left[1+\exp \left(-4 z / L_{0}\right)\right]\left(M_{0,1}^{2}+3\right)}
$$

where $z$ is the axial coordinate in the direction of the flow and $L_{0}$ is the shock thickness as defined by Prandtl [23]

$$
L_{0}=\frac{\rho_{0}(+\infty)-\rho_{0}(-\infty)}{\left|\partial \rho_{0} / \partial z\right|}
$$

The variation of the temperature and the velocity, through the shock front are deduced from the jump relations (equations (3) and (4)) using the expression (6). Muckenfuss [25] and Glansdorff [26] found that the shock thickness depends very much on the interaction potential of the governing particles. In several experiments the shock front thickness and shape were measured for argon and were in excellent agreement with the theory of Mott-Smith [27-29].

The structure of a shock front in a plasma has a number of interesting features, compared with that of a perfect gas as discussed above. These features are related to the slow energy exchange between the electrons and the heavy particles, the high electron mobility, the presence of space charges and, due to this, the presence of an electric field. Another aspect of a shock front in a plasma is the presence of recombination processes. Since the residence time of the plasma in the shock is small compared to the time needed for substantial recombination or ionization, the change in ionization degree is small. Hence, the influence of recombination processes is mainly in the heating of the electrons as in the case of the expansion [18]. As was discussed in the previous subsection, recombination processes have a small effect on the mass balances. This means that if the small heat exchange between electrons and neutral particles is neglected, the electrons behave independently from the neutral particle component, for the temperatures in the plasma jet considered [8]. On the other hand, as will be shown, the motion of the ions is influenced by the presence of electrons due to the generation of electric fields.

Since the collision times for ion-neutral and neutral-ion interactions in the shock front are much longer than the residence time in the shock and much longer than the neutral-neutral and ion-ion collision times [8], the neutral particles behave in the shock front independently from the ions and, to a good approximation, adiabatically. This means that the results for the neutral gas shock front discussed above still hold. Thus, for the neutral component the jump relations (equations (3) and (4)) are still applicable. The definition of the Mach number in equation (6), in the case of the neutral particle shock front in the present situation, is still equal to that of the neutral gas equation (5).

As stated, the behaviour of the ions in the shock front is independent from the neutral particles. However, the ions do not behave independently from the electrons. The ion shock structure is therefore not adiabatic but is influenced by the presence of the electrons. Since in the shock front the electron density increases, the electrons, due to their higher mobility, leak out of the shock front, causing an excess of negative charge density ahead of the shock front. This excess of negative charge generates an electric field. The influence of this generated electric field on the jump relations for the ion shock, can be determined by considering the electron-ion fluid as undergoing an adiabatic shock transition. As mentioned before, the neutral-ion friction can be neglected in the present approximation, because this friction is smaller than the 
corresponding pressure terms in the ion momentum equation.

The conservation laws for the electron-ion fluid for a normal adiabatic shock front are

$$
\begin{gathered}
n_{\mathrm{i}, 1} w_{\mathrm{i}, \mathrm{i}}=n_{\mathrm{i}, 2} w_{\mathrm{i}, 2} \\
m_{\mathrm{h}} n_{\mathrm{i}, 1} w_{\mathrm{i}, 1}^{2}+p_{\mathrm{i}, 1}+p_{\mathrm{e}, 1}=m_{\mathrm{h}} n_{\mathrm{i}, 2} w_{\mathrm{i}, 2}^{2}+p_{\mathrm{i}, 2}+p_{\mathrm{e}, 2} \\
\frac{1}{2} w_{\mathrm{i}, 1}^{2}+\frac{5}{2} \mathscr{R}\left(T_{\mathrm{i}, 1}+T_{\mathrm{e}, 1}\right)=\frac{1}{2} w_{i, 2}^{2}+\frac{5}{2} \mathscr{R}\left(T_{\mathrm{i}, 2}+T_{\mathrm{e}, 2}\right)
\end{gathered}
$$

where $w_{i}$ and $T_{\mathrm{i}}$ are the velocity and temperature of the ion fluid. In equations (8)-(10) terms proportional to the electron mass are neglected and from (8)-(10) the jump relations for the electron-ion fluid are easily determined and are given by

$$
\begin{gathered}
\frac{n_{\mathrm{i}, 2}}{n_{\mathrm{i}, 1}}=\frac{w_{\mathrm{i}, 1}}{w_{\mathrm{i}, 2}}=\frac{4 \mathscr{H}_{1}^{2}}{\mathscr{H}_{1}^{2}+3} \\
\frac{T_{\mathrm{i}, 2}+T_{\mathrm{e}, 2}}{T_{\mathrm{i}, 1}+T_{\mathrm{e}, 1}}=\frac{\left(5 \mathscr{H}_{1}^{2}-1\right)\left(\mathscr{H}_{1}^{2}+3\right)}{16 \mathscr{H}_{1}^{2}}
\end{gathered}
$$

where $\mathscr{A}_{1}$ is the electron-ion Mach number ahead of the ion shock front. $\mathscr{A}$ is defined as

$$
\mathscr{H}=\frac{w_{\mathrm{i}}}{\sqrt{\frac{5}{3} \mathscr{R}\left(T_{\mathrm{c}}+T_{\mathrm{i}}\right)}} .
$$

Equations (11) and (12) are identical to the jump relations for the neutral gas (equations (3) and (4)), if $T_{0}$ and $\rho_{0}$ are replaced by $T_{i}+T_{e}$ and $2 n_{\mathrm{e}}$ respectively. Using the observation that the electron temperature is approximately constant in the ion shock region (see figures 2 and 3), the jump relations for the ion gas can be determined explicitly in terms of the ion Mach number

$$
M_{\mathrm{i}}=\frac{w_{\mathrm{i}}}{\sqrt{\frac{5}{3} \mathscr{R} T_{\mathrm{i}}}}
$$

and the ratio $T_{\mathrm{e}} / T_{\mathrm{i}, 1}$. These jump relations read

$$
\begin{gathered}
\frac{n_{\mathrm{i}, 2}}{n_{\mathrm{i}, 1}}=\frac{w_{\mathrm{i}, 1}}{w_{\mathrm{i}, 2}}=\frac{4 M_{\mathrm{i}, \mathrm{l}}^{2}}{M_{\mathrm{i}, 1}^{2}+3\left(1+T_{\mathrm{e}} / T_{\mathrm{i}, 1}\right)} \\
\frac{T_{\mathrm{i}, 2}}{T_{\mathrm{i}, \mathrm{l}}}=\frac{\left[5 M_{\mathrm{i}, 1}^{2}-\left(1+T_{\mathrm{e}} / T_{\mathrm{i}, \mathrm{l}}\right)\right]\left[M_{\mathrm{i}, 1}^{2}+3\left(1+T_{\mathrm{e}} / T_{\mathrm{i}, 1}\right)\right]}{16 M_{\mathrm{i}, 1}^{2}}-\frac{T_{\mathrm{e}}}{T_{\mathrm{i}, 1}}
\end{gathered}
$$

As a result of equations (15) and (16) the compression of the ions is smaller than in absence of the electrons. The reason for this smaller compression of the ion gas is that the energy needed to compress the electrons is delivered by the ions through the generation of an electric field. In other words, the compression of the ions is smaller since they are hindered by an excess of positive charges in the ion shock front due to the mentioned leakage of the electrons to positions ahead of the ion shock front.

The situation described in the previous paragraph, the motion of the electron-ion gas through the neutral particle gas is, for the electron-ion gas, identical to the situation of a shock front in a fully ionized plasma. The shock front structure in a fully ionized gas has been determined '[30-34] and all references included the electric field due to the charge separation except the work of Jukes [34]. In the work of Jaffrin and Probstein [30], the different motion of the electrons results in a charge separation on the scale of the Debye length. The potential distribution has a double-layer shape in the ion shock region with a negative sign ahead of the ion shock front. The influence of a possible current density $j$ in the shock front region was investigated by Greenberg et al [31]. However, they did not include viscosity, which caused a discontinuous solution, but did demonstrate that large electric fields can exist $\left(4 \times 10^{6} \mathrm{~V} \mathrm{~m}^{-1}\right.$ !) in the ion shock front.

For $p=40$ and $13.3 \mathrm{~Pa}$ (see figure 2), the ratio between the neutral particle density ahead and behind the shock front is approximately four, indicating that the neutral particle Mach number is large (see equation (3)). For the ion density the situation is different. Note the overshoot of the ion density for higher background pressures caused by deceleration and cooling of the plasma [35]. It can be concluded that for lower background pressures the jump in the ion density decreases. Again this confirms the fact that the ions and neutral particles behave independently in the shock front. If the shock transition of the ions would be gas dynamic in nature, a smaller density jump would mean a smaller ion Mach number. However, as discussed, the motion of the ions in the shock front is influenced by the presence of electrons effectively causing a smaller than gas dynamic ion density jump.

In figure 7 the normalized shock fronts are given for the settings in table 1 . The Mott-Smith relation [24] (equation (6)) is used to normalize the neutral particle and ion shock fronts, each with their own shock strengths (respectively $S_{0}$ and $S_{1}$ ) and shock thicknesses, respectively $L_{i}$ and $L_{0}$. The shock strength for the neutral particles is given by (see equation (6))

$$
S_{0}=\frac{\left(M_{0,1}^{2}-1\right)}{\left(M_{0,1}^{2}+3\right)}
$$

For the ion shock a similar relation holds, with the ion-electron Mach number $\mathscr{A}_{\mathrm{l}}$. Although the accuracy of the measured neutral densities for figure $7(a)$ is limited, a clear correspondence with the Mott-Smith (see equation (6)) solution is seen. For the ion shock front, figure $7(b)$, the agreement with the result (equation (6)) of Mott-Smith is even better. Here it should be remembered that the ion density has a small overshoot at the end of the ion shock front. Therefore the data at the right in figure 7 have a larger uncertainty. In figure 8 the parameters $L_{0}$ and $L_{\mathrm{j}}$ and the Mach numbers $M_{0,1}$ and $\mathscr{H}_{1}$ determined from the fit of equation (6) are shown as a function of the vessel pressure for the first three settings of table 1 . In table 3 the results of the fit of equation (6), including an estimation of the error in the determined parameters, are given for the five settings of table 1 .

As can be concluded from table 3, the Mach number $M_{0.1}$ is always larger than the Mach number $\mathscr{H}_{1}$. This 
(a)

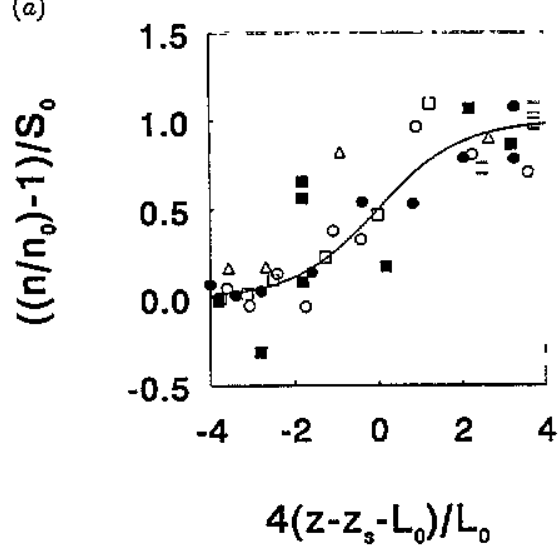

(b)

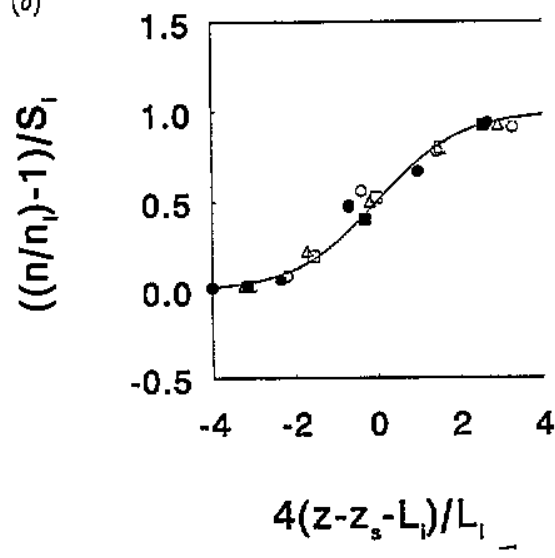

Figure 7. The normalized shock fronts of the neutral particles (a) and the ions (b) (see table 1): (1) , (2) (3) $\triangle$, (4) $\square$ and (5) $\bigcirc$. The line is the Mott-Smith relation (equation (6)).

is in agreement with equation (15), where it was shown that the presence of electrons decreases the ion density jump. Another important conclusion from figure 8 is that the neutral particle Mach number is larger for lower background pressure, whereas for the ion shock front the situation is reversed. In both cases, however, the shock thickness decreases for increasing background pressure. This is explained by the increasing densities and thus decreasing collision times for ion-ion and neutral-neutral interactions.

The Mach number $\mathscr{M}_{1}$ decreases as the background pressure decreases, which is opposite to the behaviour of the neutral particles. The electron temperature appearing in $\mathscr{H}_{1}$ (see equation (13)) becomes smaller for smaller background pressures which should lead to a smaller $\mathscr{M}_{1}$. Therefore there are only two possibilities which lead to a larger Mach number. The first is that the velocity of the ions for lower background pressure decreases more during the expansion than for higher background pressures. This is related to the structure of the electric field in the expansion. For example, a deceleration of the ions in the neighbourhood of the shock
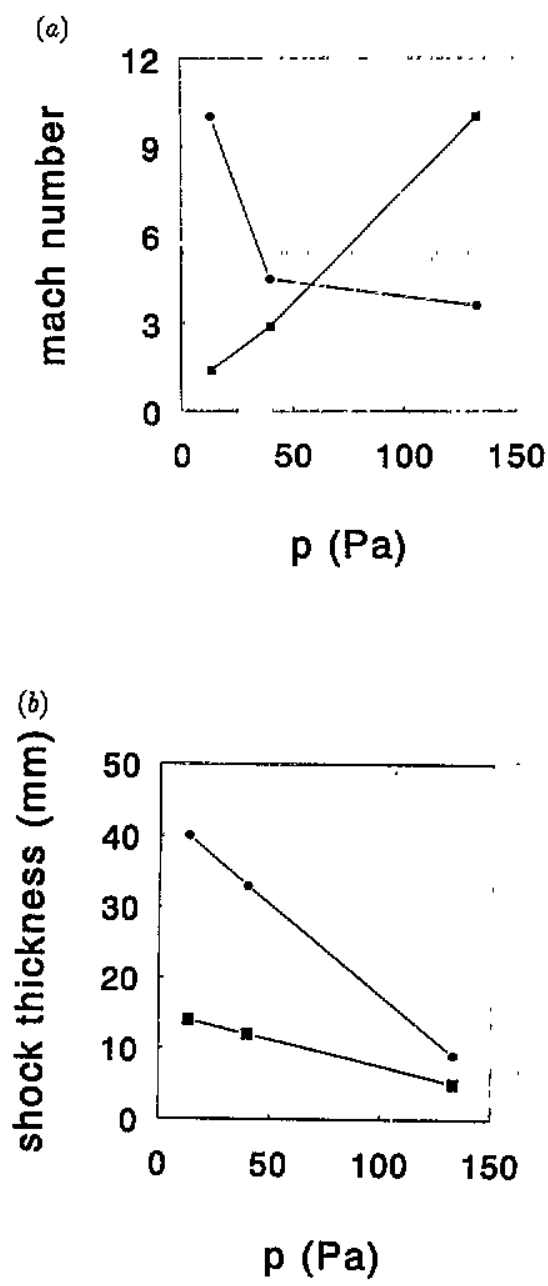

Figure 8. The Mach number and the shock thickness as function of the vessel pressure for the neutral particles and the ions (O) and the ions ( $\square$ ). The lines are drawn to guide the eye.

front is caused by a positive space charge behind the shock front [8]. A second reason could be a higher temperature of the ions.

If the pressure behind the shock front is equal to the background pressure, the velocities and temperatures in front of the shock and behind the shock can be determined as follows. From the results of table 3 , together with the jump relations for the temperature (equation

Table 3. The parameters $M_{0,3}, L_{0}, \|_{1}, L_{0}$ determined from the shock front structure of the neutral particles and the ions for the different settings of table 1 .

\begin{tabular}{lllll}
\hline Settings & $M_{0,1}$ & $L_{0}(\mathrm{~mm})$ & $\mathscr{H}_{1}$ & $L_{i}(\mathrm{~mm})$ \\
\hline 1 & $4.5 \pm 0.3$ & $33 \pm 2$ & $2.9 \pm 0.3$ & $12 \pm 1$ \\
2 & $10 \pm 2.0$ & $40 \pm 3$ & $1.4 \pm 0.1$ & $14 \pm 1$ \\
3 & $3.6 \pm 0.3$ & $9 \pm 0.5$ & $>10$ & $5 \pm 0.5$ \\
4 & $8.0 \pm 1.0$ & $32 \pm 2$ & $2.8 \pm 0.3$ & $13 \pm 1$ \\
5 & $3.8 \pm 0.3$ & $30 \pm 2$ & $2.0 \pm 0.3$ & $11 \pm 1$ \\
\hline
\end{tabular}


(4) for the neutral particles and equation (12) for the ions), the ratio of the temperature ahead and behind the shock front is easily determined. From the background pressure, together with the values of the ion and neutral particle densities and the electron temperature behind the shock front (which is approximately equal to the electron temperature ahead of the shock front), the heavy particle temperature is determined. The results for the neutral particles and ions are given in tables 4 and 5 .

In the calculation of the temperatures and velocities it is assumed that the shock front is normal to the direction of the flow. The parameters in tables 4 and 5 are not corrected for the shock front curvature [36], since these corrections are much smaller than the errors in the determined parameters $( \pm 10$ to $30 \%)$. From tables 4 and 5 it can be concluded that the ions and neutral particles just ahead of the shock behave differently, which is reflected in a different velocity and temperature for the ions compared with the neutral particles. Furthermore, the velocities and temperatures are higher ahead of the shock front. This is in agreement with the lower jump of the ion density. The velocity and temperature of the ions for the high background pressure ahead of the ion shock front are unphysical and related to the indeterminacy of the Mach number $\mathscr{H}_{1}$ (see table 3). The values of the neutral particle temperature for setting 1 of table 1 are in agreement with the measurements of Meulenbroeks et al [37]. The values of the determined neutral particle velocity are similar to the results of Poissant and Dudeck [16] and Kroesen et al [6].

Table 4. The neutral particle temperature and velocity ahead and behind the shock front determined from the jump relations for the different settings of table 1 . It is assumed that the pressure behind the shock front equals the vessel pressure and that $T_{0,2}=T_{\mathrm{i}, 2}$.

\begin{tabular}{lllll}
\hline Settings & $T_{0,2}(\mathrm{~K})$ & $w_{0,2}\left(\mathrm{~m} \mathrm{~s}^{-1}\right)$ & $T_{0,1}(\mathrm{~K})$ & $w_{0,1}\left(\mathrm{~m} \mathrm{~s}^{-1}\right)$ \\
\hline 1 & 3700 & 550 & 510 & 1900 \\
2 & 6000 & 670 & 190 & 2800 \\
3 & 3600 & 550 & 730 & 1800 \\
4 & 4400 & 550 & 210 & 2100 \\
5 & 6900 & 750 & 1300 & 2500 \\
\hline
\end{tabular}

Table 5. The ion temperature and velocity ahead and behind the shock front determined from the jump relations for the different settings of table 1 . It is assumed that the pressure behind the shock front equals the vessel pressure and that $T_{0,2}=T_{i, 2}$. Furthermore, the value of the electron temperature behind the ion is used.

\begin{tabular}{lllll}
\hline Settings & $T_{\mathrm{i}, 2}(\mathrm{~K})$ & $w_{1,2}\left(\mathrm{~m} \mathrm{~s}^{-1}\right)$ & $T_{\mathrm{i}, 1}(\mathrm{~K})$ & $w_{1,1}\left(\mathrm{~m} \mathrm{~s}^{-1}\right)$ \\
\hline 1 & 3700 & 940 & 1230 & 3370 \\
2 & 6000 & 1140 & 5000 & 2340 \\
3 & 3600 & 2900 & $(11600)$ & $(130)$ \\
4 & 4400 & 930 & 1490 & 3240 \\
5 & 6900 & 1080 & 3060 & 3700 \\
\hline
\end{tabular}

\section{Conclusions}

In conclusion, several features of a plasma expanding from a cascaded arc were discussed. The expansion of the plasma in the region ahead of the shock front is independent of the vessel pressure whereas the shock front is closer to the exit of the cascaded arc if the vessel pressure increases. The behaviour of the ions and neutral particles in the supersonic expansion is near to adiabatic. The adiabatic behaviour is confirmed by comparing the ion and neutral particle density with the model of Ashkenas and Sherman [12]. Furthermore, three-particle recombination has a minor effect on the ion and neutral particle density in the expansion. The ions and the neutral particles behave independently in the shock front. The shock thickness of the ion shock front is systematically smaller than that of the neutral particles which is related to the larger viscosity of the ions. In the description of the ion shock front the influence of the electron has to be accounted for. This results in a smaller shock strength of the ions since they deliver the energy for the compression of the electrons. For both the ion and the neutral particle shock fronts a good agreement is found with the adiabatic model of Mott-Smith (equation (6)). An interesting observation is the fact that the electron temperature increases ahead of the density shock fronts. A subsequent paper is devoted to this subject [10].

\section{Acknowledgment}

We would like to thank $M J F$ van de Sande, $H$ M M de Jong and A B M Hüsken for their skillful technical assistance. We thank J A M van der Mullen for helpful discussions. The research of $M C M$ van de Sanden has been made possible by a fellowship of The Royal Netherlands Academy of Arts and Sciences.

\section{References}

[1] Beulens J J, Buuron A J M and Schram D C 1991 Surf. Coatings Technol 47401

[2] Buuron A J M, Beulens J J, van de Sande M J F, Schram D C and van der Laan J G 1991 Fusion Technol. 19 2049

[3] Meeusen G J, Ershov-Pavlov E A., Meulenbroeks R F G, van de Sanden M C M and Schram D C 1992 J. Appl. Phys. 714156

[4] de Graaf M J, Severens R, Dahiya R P, van de Sanden M C M and Schram D C 1993 Phys. Rev. E 482098

[5] Ruyten W M and Keefer D 1992 Appl. Phys. Lett. 61880

[6] Kroesen G M W, Schram D C and de Haas J C M 1990 Plasma Chem. Plasma Proc. 10531

[7] van de Sanden $M C M$, de Regt J M, Janssen $G M$, Schram D C, van der Mullen J A M and van der Sijde B 1992 Rev. Sci. Instrum. 633369

[8] van de Sanden M C M $1991 \mathrm{PhD}$ thesis University of Technology of Eindhoven, The Netherlands

[9] Beulens J J, Milojevic D, Schram D C and Vallinga P M 1991 Phys. Fluids B 32548 
[10] van de Sanden M C M, van den Bercken $R$ and Schram D C 1994 Plasma Sources Sci. Technol. 3511

[11] Young W S 1975 Phys. Fluids 181421

[12] Ashkenas H and Sherman F S 1966 Rarefied Gasdynamics vol 4 ed J H de Leeuw (New York: Academic)

[13] Fraser R B, Robben F and Talbot L 1971 Phys. Fluids 14 2317

[14] Kirchhoff R H and Talbot L 1971 AIAA J. 91098

[15] Jenkins R C 1971 AIAA J. 91383

[16] Poissant G and Dudeck M 1985 J. Appl. Phys. 581772

[17] Kobayashi H, Nakagawa T and Nishida M 1984 Rarefied Gasdynamics vol 14 ed H Oguchi (Tokyo: University of Tokyo Press)

[18] van de Sanden M C M, de Regt J M and Schram D C 1993 Phys. Rev. E 472792

[19] Biberman L M, Vorob'ev V S and Yakubov I T 1987 Kinetics of Nonequilibrium Low Temperature Plasmas (New York: Plenum)

[20] Hinnov E and Hirschberg J G 1962 Phys. Rev. 125795

[21] Benoy D, van der Mullen J A M, van de Sanden M C M, van der Sijde B and Schram D C 1993 J. Quant. Spectrosc. Radiat. Transfer 49129

[22] Landau L and Liftshitz E 1989 Fluid Mechanics (London: Pergamon)
[23] Zel'dovich Y B and Raizer Y P 1966 Physics of Shock Waves and High Temperature Hydrodynamic Phenomena vols 1 \& 2 (New York: Academic)

[24] Mott-Smith H M 1951 Phys. Rev. 82885

[25] Muckenfuss C 1960 Phys. Fluids 3320

[26] Glansdorff P 1962 Phys. Fluids 5371

[27] Camac M 1966 Proc. Rarefied Gasdynamics vol 4 ed J H de Leeuw (New York: Academic)

[28] Russell D A 1966 Proc. Rarefied Gasdynamics vol 4 ed J H de Leeuw (New York: Academic)

[29] Alsmeyer H 1976 J. Fluid Mech. 74497

[30] Jaffrin M Y and Probstein R P 1964 Phys. Fluids 101658

[31] Greenberg O W. Sen H K and Treve Y M 1960 Phys. Fluids 3379

[32] Imshennik V S 1962 Sov. Phys.-JETP 15167

[33] Shafranov V D 1957 Sov. Phys.-JETP 51183

[34] Jukes J D 1957 J. Fluid Mech. 3275

[35] Kroesen G M W, Schram D C and de Haas J C M 1990 Plasma Chem. Plasma Proc. 10531

[36] Robben F, Kunkel W B and Talbot L Phys. Rev. 132 2363

[37] Meulenbroeks R F G, van der Heijden P A A, van de Sanden M C M and Schram D C 1994 J. Appl. Phys. 752775 